\title{
Molecular dynamics modeling of multicolor FRET-experiment
}

Andryushchenko V.A.

Institute of Thermophysics, SB RAS, Novosibirsk, Russia

*e-mail: vladimir.andryushchenko@gmail.com

Key words: protein folding, FRET spectroscopy, molecular dynamics, free energy surfaces

Motivation and Aim: The Förster resonance energy transfer (FRET) method has become a powerful tool for studying protein dynamics. Currently, the donor and acceptor used in experiments usually attached to the ends of the protein chain [1]. Results commonly presented as a one-dimensional (1D) free energy profile. However, since protein dynamics are very complex, such $1 \mathrm{D}$ profiles do not provide a sufficiently detailed description of the styling process. At the same time, the donor and acceptor can be attached not only to the ends of the protein but to the inner parts of the protein chain (a multicolor experiment) [2]. In this case, two-dimensional free energy surfaces (FES) can be constructed that provide incomparably richer information about the folding process than 1D profiles [3]. Naturally, the question arises as to the optimal arrangement of the minimum amount of fluorophores in order to obtain the best possible information on protein folding.

Methods and Algorithms: Since the main goal was to understand the situation in general, a coarse-grained protein representation was used, i.e., a bead placed at the position of the $\mathrm{C} \alpha$-atom represented each protein residue. The simulations performed with molecular dynamics methods. Selected residues (beads) represented the donor and acceptor. Two fluorophores attached to the ends of the protein and another inside the protein chain. The site of attachment of the last fluorophore was changing.

Results: The BBL domain protein folding has been modeling for different locations of the fluorophores along the protein chain. For all cases, two-dimensional free-energy surfaces and radial distribution functions, characterizing the relative position of the fluorophores relative to each other and the protein chain were constructed. On the base of the obtained data, the optimal location of fluorophores was determined, which made possible to reproduce the characteristic states of a protein in the space of distances between fluorophores.

Conclusion: The data available in the experiments i.e. distances between fluorophores (with a suitable arrangement of the three fluorophores) is enough to recover information on protein states similar to the information available in the modeling of protein folding in "usual variables", such as the radius of gyration and RMSD.

Acknowledgements: Supported by the RFBR (18-04-00013).

\section{References}

1. Neuweiler H., Johnson C.M., Fersht A.R. Direct observation of ultrafast folding and denatured state dynamics in single protein molecules. PNAS. 2009;106(44):18569-18574.

2. Lerner E. et al. Toward dynamic structural biology: Two decades of single-molecule Förster resonance energy transfer. Science. 2018;359(6373):eaan1133.

3. Andryushchenko V.A., Chekmarev S.F. Modeling of multicolor single-molecule forster resonance energy-transfer experiments on protein folding. Journal Physical Chemistry B. 2018;122(47):1067810685. 